\title{
Pariser Chirurgie von 1794 bis 1850
}

\author{
Von Erwin H. Ackerknecht, Zürich
}

Vor zwei Jahren habe ich hier über die Therapie der Pariser Internisten in der «klassischen» Periode berichtet ${ }^{1}$. Seither hat Frau Dr. Erna LesKY ${ }^{2}$ ungemein lichtvoll über ähnliche Probleme im Wien der gleichen Zeitperiode gehandelt. Sie hat von vornherein Chirurgie und Geburtshilfe in ihre Betrachtung einbezogen. Im Anschluß daran möchte ich hier nun auch einen kurzen Überblick über die Chirurgie des «klassischen» Zeitabschnitts in Paris geben, die sich allerdings stark von der Wiens unterscheidet, auch insofern, als die Einheitlichkeit der Orientierung der drei therapeutischen Branchen in Paris viel geringer ist. Das liegt wohl zum Teil daran, daß, während in Wien im 18. Jahrhundert die innere Medizin überragend, die Chirurgie aber unbedeutend gewesen, es in Paris umgekehrt gegangen war: Die innere Medizin war unbeachtet, die Chirurgie aber führend gewesen; ja, gerade hier hatte sich ihre soziale und wissenschaftliche Rehabilitation vollzogen. Die Chirurgie hatte darum die dann so glänzende Pariser innere Medizin des 19. Jahrhunderts geformt. WunderLICH hat das schon ganz eindeutig festgestellt: «In der Tat ist die ganze Richtung der neueren französischen Medizin der chirurgischen Schule entsprungen. ${ }^{3}$

Temкin hat in neuerer Zeit wieder auf diesen Sachverhalt hingewiesen ${ }^{4}$. Tatsächlich waren ja nicht nur Bichat, sondern auch Laenneg, Broussais, Récamier, Cruveilhier und Chaussier zuerst als Chirurgen ausgebildet. Auch hatten nicht nur Laenneg und Bouillaud, sondern sogar Pinel und Broussars versichert, daß sie die inneren Krankheiten wie die Chirurgen die äußeren angehen würden ${ }^{5}$. Selbst der Neohumoralismus der späteren Pariser Internisten wie ANdral begann 1824 mit Velpeau und anderen Chirurgen ${ }^{6}$.

${ }^{1}$ Die Therapie der Pariser Kliniker zwischen 1795 und 1840, Gesnerus 15 (1958) 151-63.

${ }^{2}$ Von den Ursprüngen des therapeutischen Nihilismus, Sudhoffs Archiv 44 (1960) 1-20.

3 C. A. Wunderlich, Geschichte der Medizin, Stuttgart 1859, S. 244.

${ }^{4}$ O. Temkin, The Role of Surgery in the Rise of Modern Medical Thought, Bull. Hist. Med. 25 (1951) 248-59.

5 E. H. Ackerknecht, La médecine à Paris entre 1800 et 1850, Paris 1958, p. 17; id., Broussais, Bull. Hist. Med. 27 (1953) 321.

${ }^{6}$ Denonvilliers, Nélaton, Velpeau, Félix Guyon et Léon Labbé. Rapport sur les progrès de la chirurgie, Paris 1867, S. 7. 
Im Gegensatz zur Pariser inneren Medizin des 18. Jahrhunderts war die Chirurgie mit ihrer Académie weltberühmt gewesen ${ }^{7}$. Zahlreiche Ausdrücke unserer Fachsprache wie Tourniquet, Troquart stammen aus dieser Zeit. Allerdings war selbst die Académie de Chirurgie, wenigstens nach Ansicht von Desault, schließlich auch nicht von der allgemeinen Fäulnis frei geblieben ${ }^{8}$. Trotzdem war in der Chirurgie keine so tiefgehende Revolution notwendig gewesen als in der inneren Medizin, man konnte eher ans Gegebene anknüpfen, fortsetzen, konservativer sein. Kein Internist hätte gewagt, 1814 über die Medizin zu sagen, was der Chirurg A.Boyer mit großer Ruhe bezüglich der Chirurgie an den Anfang seines berühmten Traité des maladies chirurgicales setzte: "La chirurgie a fait de nos jours les plus grands progrès, et semble avoir atteint, ou peu s'en faut, le plus haut degré de perfection dont elle paraisse susceptible.»

Selbst der letzte große Chirurg der alten Schule, der gleichzeitig der erste große Chirurg der neuen war und als kühner Neuerer galt, P.J.Desault (1744-1795), ist eher konservativ zu nennen. Seine radikalste Neuerung war wohl die Einführung des klinischen Unterrichts. Schon seine vielgerühmte Beschäftigung mit topographischer Anatomie war nichts gänzlich Neues und wurde dann von Roux, BÉclard und VelPEau glänzend weiterentwickelt. Wie intensiv die Pariser Chirurgen dieser Periode an der Leiche studierten, illustriert die Tatsache, daß Lisfranc in seinen Operationskursen jährlich etwa tausend Leichen verbrauchte ${ }^{9}$. Die pathologische Anatomie wurde erst von Desaults Schüler Bichat und dessen Schülern Dupuytren, Bayle und Laennec gründlich gepflegt.

Sonst beschäftigte sich Desault mit traditionellen Aufgaben, wie z.B. der Amputation. Diese beschränkte er so sehr wie möglich, und eine Parallele zur abwartend konservativen skeptischen Haltung der ConvisarT und Pinel ist deutlich sichtbar. Auch die Frakturbehandlung, in der Desault so glänzte, oder Blasensteinoperation oder Arterienligatur bei Aneurysma waren alte chirurgische Themen. Es sollte übrigens betont werden, daß Desault nicht nur Amputationen, sondern auch Trepanationen zu vermeiden suchte, die Unzahl der Mittel beschränkte und das Aneurysma eher durch Kompression als durch Operation zu heilen suchte.

${ }^{7}$ Georg Fischer, Chirurgie vor 100 Jahren, Leipzig 1876. De Fourmestraux, Histoire de la chirurgie française 1790-1920, Paris 1934. P. Delaunay, Le monde médical parisien au $18^{e}$ siècle, Paris 1906.

${ }^{8} \mathrm{~J}$. RochaRD, Histoire de la chirurgie française au $19^{e}$ siècle, Paris 1875, S. 3.

${ }^{9}$ F. A. Аммок, Parallele der französischen und deutschen Chirurgie, Leipzig 1823, S. 39. 
Seine zurückhaltende Einstellung hätte sich wahrscheinlich noch stärker durchgesetzt, wenn Frankreich nicht gerade in den ersten zwanzig Jahren der neuen Schule ununterbrochen Krieg geführt hätte. Dieser Umstand stärkte einerseits die Chirurgie im allgemeinen, brachte sie andererseits weitgehend unter den Einfluß der Armeechirurgen, und das um so mehr, als Frankreich sich seit Henri IV mehr als jedes andere Land um diesen Zweig der Chirurgie bemüht hatte. Die Militärchirurgen wurden durch die Umstände dazu gedrängt, auf die unzähligen Wundsalben der Privatpraxis zu verzichten und diese lang vor voN KERN durch das Wasser zu ersetzen (z.B. Percy 1785). Im Universitätsmilieu wurde das Wasser dann erst später stark durch BÉRARd JEune und Josse (1835) propagiert.

Dieselben Notstände des Lagerlebens veranlaßten die französischen Militärchirurgen zu einer drastischen und segensreichen Reduktion in der Zahl der Instrumente und Verbände, die darum, wie AmmoN ${ }^{10}$ angibt, in den zwanziger Jahren in Frankreich im Gegensatz zu Deutschland allgemein vorherrschte. Zurückhaltend waren die Armeechirurgen auch noch in der Frage des débridement.

Aber auf dem Gebiete der Amputation mußten sie aktiv sein, und zwar unverzüglich (amputation immédiate). Sie benützten zur Blutstillung die Ligatur und nähten im allgemeinen die Wunde sofort im Hinblick auf eine Heilung per primam intentionem. Roux hatte darum keine besonderen Schwierigkeiten, diese Technik nach seiner berühmten Studienreise nach England im Jahr $1814^{11}$ auch in der Zivilpraxis einzuführen, während sie in Deutschland verpönt blieb ${ }^{12}$. Wenn man von der französischen Militärchirurgie dieser Jahre spricht, ist es unmöglich, nicht wenigstens auch auf die Erfindung der Ambulanzen hinzuweisen und die Namen ihrer Erfinder, der zwei führenden Armeechirurgen der Zeit, zu erwähnen: P.F.Percy (1754-1825), der seine Karriere der Förderung durch A.Louis verdankte, und J.D.LARrey (1766-1842), der an 25 Feldzügen, 60 Schlachten, 400 Gefechten teilgenommen hatte. Er soll bei Borodino in 24. Stunden 200 Amputationen ausgeführt haben. Auch im Frieden blieb er wegweisend, z.B. auf dem Gebiet der Rhinoplastik.

Der trotz der militärischen Einflüsse verhältnismäßig konservative Charakter der französischen Chirurgie ist sogar bei G. DUPUYTREN (1777-1835),

10 id., S. 74.

11 PH. J. Roux, Relation d'un voyage fait à Londres en 1814, Paris 1815.

12 Ammon, l. c. S. 118. 
der von 1815 bis 1835 der Diktator oder «Napoleon» der französischen Chirurgie war, ganz deutlich, besonders wenn man ihn mit dem zur selben Zeit die innere Medizin tyrannisierenden BroussaIs vergleicht ${ }^{13}$. Von diesem übernahm DupuYtren übrigens leider die verschwenderische Anwendung von Blutegeln, die Hungerdiät und die Kataplasmen. Die von ihm geübte chirurgische Behandlung von Hernien, Hydrocoelen, Star, Fisteln, Blasensteinen oder Aneurysmen sind wieder durchaus traditionelle Themen. Die Luftembolie war schon vor ihm, d.h. 1818, von BEAUCHÊNE beobachtet worden. Die noch heute nach ihm benannte Kontraktur ist nicht sehr beträchtlich. Trotzdem hat auch er wirklich Neues geschaffen, wie wir im folgenden sehen werden. Denn die Pariser Schule schuf trotz aller Hemmungen auch auf chirurgischem Gebiet Neues.

Die gewaltigste Neuleistung waren zweifellos die Generalisierung des klinischen Unterrichts und die Vereinigung der seit Jahrhunderten getrennten Medizin und Chirurgie in den Ecoles de Santé von 1794 und die Verteidigung dieser Einheit gegen die Attacken der Reaktion nach 1815. Um diese neue Situation zu symbolisieren, nannte LAssus, einer der ersten zwölf Professoren der neuen Pariser Ecole de Santé, sein 1794 erscheinendes Werk Médecine opératoire, nicht «Chirurgie opératoire», und Sabatier, Lisfranc, Velpeau und Malgaigne folgten ihm auf diesem Weg. Es ist wohl keine Übertreibung, zu sagen, daß zu keiner andern Zeit und an keinem andern Ort die Einheit von Chirurgie und innerer Medizin so weitgehend verwirklicht worden ist wie in Frankreich in der ersten Hälfte des 19. Jahrhunderts ${ }^{14}$.

SIGERIST hat schon vor dreißig Jahren darauf hingewiesen, daß das, was wir heute Chirurgie nennen, eine sehr aggressive Disziplin, die sich vor allem in der zweiten Hälfte des 19. Jahrhunderts entwickelte und die sich sehr wesentlich von der bis dahin seit Jahrtausenden unter demselben Namen praktizierten konservativen, meist wundärztlichen Kunst unterscheidet, vor allen Dingen ein Kind des «anatomischen Gedankens», der lokalisierenden antihumoralistischen Theorie der Krankheiten war und nicht in erster Linie ein Resultat der Entdeckung von Anästhesie und Asepsis ${ }^{15}$. Dieses Aktivwerden der Chirurgie unter dem Einfluß des Lokalismus noch

\footnotetext{
${ }^{13}$ L. Delhoume, Dupuytren, Paris 1935. Auf D.s wenig schönen, persönlichen Charakter einzugehen, erübrigt sich hier.

${ }^{14}$ Siehe auch Ackerknecht, 1958, S. 17.

${ }^{15}$ H. E. Sigerist, Einführung in die Medizin, Leipzig 1931, S. 316.
} 
vor Morton und Lister können wir nun sehr schön in Paris beobachten. Die Chirurgie hatte dazu beigetragen, die Medizin auf den lokalistischen Weg zu führen. Von dem medizinischen Lokalismus erhielt sie nun ihrerseits wichtige Impulse zu aktiverem Vorgehen. Das ist das zweite Neue, das die Schule in der Chirurgie bringt.

VelPeAu zählt gleich im ersten Abschnitt seiner Nouveaux Eléments de Médecine Opératoire (1832) auf, was seiner Meinung nach seit Sabatier, d.h. seit Beginn der neuen Schule, in der operativen Chirurgie dazugekommen sei. Er erwähnt die plastischen Operationen (rhino-, cheilo-, blepharo-, oto-, bronchoplastie et staphyloraphie); die torsion et piqûre des artères; die lithotritie et cautérisation de l'urétère; die amputation de la matrice et extirpation de l'ovaire; extirpation de l'anus; plusieurs résections.

VelPEAus Liste bleibt auch heute wenig hinzuzufügen. Nur würden wir eine Diskussion derselben eher am Ende, mit den Resektionen von Knochen und Gelenken, anfangen, da zeitlich hier in Frankreich der erste Schritt zu einer aktiveren und doch auch wieder vorsichtigen Chirurgie getan wurde. Die Priorität gebührt zweifellos den Engländern (Charles White, 1768). Doch war der einfache Landarzt, von dem die neue Technik der Gelenkresektion in Frankreich 1782 ausging, MoREAU aus Bar-sur-Ornain, durchaus originell, und seine Erfindung verbreitete sich nach einem zufälligen Zusammentreffen mit Percy (1792) schnell in der Armee. Auch die Zivilchirurgie glänzte auf diesem Gebiet. Seine Unterkieferresektion (1812) war vielleicht Dupurtrens beste Neuschöpfung, während auf dem Gebiet der Gefäßunterbindung wegen Aneurysma er wie Roux mehr oder weniger Epigonen der Angelsachsen waren.

Am zukunftsträchtigsten waren zweifellos jene Operationen, die als Anfänge der Abdominalchirurgie gewertet werden können. Da ist vor allem die Exstirpation des krebsigen Rectum durch J.Lisfranc (1790-1847) zu nennen und die Anlegung eines künstlichen Anus praeternaturalis durch Amussat 1839, sowie SÉdillots Gastrostomie von 1846. Dupuytren hatte zur Behandlung des spontanen anus praeter das Enterotom erfunden (1828). Jobert de Lamballe (1824) und Lembert (1826) hatten die Technik der Darmnaht außerordentlich verbessert.

Auf dem Gebiet der Uteruschirurgie - Dechambres Dictionnaire prophezeite noch 1876, sie werde wieder verschwinden ${ }^{16}$ - hatte derselbe Lis-

${ }^{16}$ Dechambre, Dictionnaire Encyclopédic des sciences médicales, Band 16 (1876) S. 434, Über RÉcamiers Gynäkologie s. P. TriaIre, Récamier, Paris 1899. 
FRANC, der heute nur noch für seine Fußamputation bekannt ist, nach 1825 die Cervixamputation zur Routine erhoben. Er mußte sich übrigens von PAUly vorwerfen lassen, er habe seine Resultate frisiert ${ }^{17}$. Derselbe Vorwurf wurde auch gegen DupuYtren erhoben und bei Jules GuÉrin nachgewiesen ${ }^{18}$. Im Jahr 1829 wurde von J.C. RÉCAMIER (1774-1842) sogar die Totalexstirpation des Uterus durchgeführt. Dieser «Internist» hatte auch seit 1801 das Speculum wieder eingeführt und die Curette erfunden. Er ist nicht der einzige Internist, der als Chirurg glänzte; siehe z. B. auch LALLEMaND. Andererseits betätigten sich Chirurgen internistisch; DupuYtren und Velpeau beschrieben z. B. die Polycythaemie.

Die urologische Betätigung ist nichts Neues in einem Land, das seit Jahrhunderten durch seine Steinschneider und Blasensteine berühmt war. Es ist aber typisch, daß um 1820 von Civiale, Leroy d'Etiolle, Amussat und Heurteloup ein unblutiges Verfahren zur Entfernung des Steins, die Lithotritie, geschaffen wurde. Auch zeigt sich bei den urologischen Chirurgen bereits deutlich die Tendenz zur Spezialitätenbildung.

Noch wichtiger als die Autoplastien, von denen Velpeau so beeindruckt war - auf diesem Gebiet war Roux führend, teils nachahmend, teils originell wie bei der Staphylorrhaphie von 1816 -, waren die neuen orthopädischen Operationen, die Teno- und Myotomien, die zur Vermeidung der Wundinfektion subkutan durchgeführt wurden. DELPECH durchschnitt 1816 zum ersten Mal subkutan die Achillessehne, Dupurtren 1822 den sternocleidomastoideus. Jules Guérin übertrieb die Tenotomie dann ins Sinnlose. Durch Delpech, der seine Autoplastien und Tenotomien als «Orthomorphie» zusammenfaßte, und seinen Nachfolger Lallemand wurde Montpellier zum zweiten großen chirurgischen Zentrum Frankreichs. Es ist typisch für den hippokratisch-konservativen Charakter dieser Orthopädie, daß nicht nur neue Operationen erfunden wurden, sondern auch die Gymnastik als Therapie von Pravaz, Lachaise, Delpech, Amoros, Récamier u.a. in großem Maßstab wiedererweckt wurde.

Der Einfluß der Chirurgie auf die innere Medizin spiegelt sich in der Einführung der Tracheotomie bei Diphtherie durch BREtonneau und seine Schule nach 1825 und in der chirurgischen Behandlung des Empyems (z.B. Fleury, 1838). Die Behandlungen von Hydrocoelen durch Jodinjektionen (Velpeau, 1835) ist ein weiterer Ausdruck der konservativen Tendenzen

${ }^{17}$ Préface de H. Pauly pour Lisfranc, Maladies de l'uterus, Paris 1836.

18 E. Pillastre, Malgaigne, Paris 1905, S. 183-201. 
in der Pariser Chirurgie. Diese wurden nach etwa zwanzig Jahren größerer Aktivität sehr stark. Kein anderer als Lisfranc, wohl einst der aktivste Chirurg der Schule, rief in seinem Précis de Médecine Opératoire von 1843 zur äußersten Mäßigung auf. VelpeaU und Magendie wendeten sich 1848 gegen zu reichliche Amputationen ${ }^{19}$. Der Grund ist klar: Man war, ohne Asepsis und Anästhesie, in eine Sackgasse geraten. Schock und Wundfieber verwandelten die technisch geglückten Operationen in traurige Niederlagen. Die Mortalitätsstatistiken bei großen Operationen, z.B. die von MaLGaIgne aus dem Jahr 1841, sind erschütternd. Von 21 Uterusexstirpationen waren 183221 während oder kurz nach der Operation gestorben ${ }^{20}$. Selbst Neuentdeckungen auf dem Gebiet der chirurgischen Pathologie wie die Appendicitis waren angesichts der operativen Ohnmacht von geringer Bedeutung.

Die zwei großen, notwendigen Neuerungen, Anästhesie und Asepsis, wurden in Paris weder gefunden noch auch nur systematisch gesucht. Die Hypnosenarkose von Jules Cloquet (1829) blieb rein episodisch. Die Wundbehandlung mit Eau de Javel durch Cullerier, die Hautprophylaxe mit Silbernitrat durch Chassaignac, die Watteverbände von Mayor und Alphonse Guérin, die réunion immédiate der Operationswunden, die subkutanen Prozeduren sind nur ein mehr oder weniger klares, tastendes Herumprobieren in Richtung der Asepsis. Der «Ecraseur» von Сн.M.E. Chassaignac (1804-1879), von dem andererseits auch die Drainage stammt, war sogar eine schlimme Entgleisung. Um zur Anästhesie und Asepsis zu gelangen, mußte man an Chemie und Mikroskopie interessiert sein. Dieses Interesse war bei den älteren Pariser Chirurgen ebensowenig vorhanden wie bei ihren internistischen Kollegen ${ }^{21}$. Erst BROCA und Follin begannen zu mikroskopieren.

Die französische Chirurgie der ersten Jahrhunderthälfte war brillant und berühmt. Sie wurde von den Chirurgen der ganzen Welt als Lehrmeisterin gesucht und hat sehr Nützliches geleistet, aber nicht Epochales wie die innere Medizin der gleichen Periode. Das ist an sich nicht überraschend. Die Chirurgie konnte wohl einen wichtigen Anstoß zur Anschauungsweise der Pinel, Bichat, Broussais und Laenneg geben, aber als therapeutische Technik konnte und sollte sie keine neue medizinische Gesamttheorie schaffen. Als therapeutische Technik selbst ist sie widerspruchsvoll. Auch

19 Denonvilliers, l. c. S. 623.

${ }^{20}$ Rochard, l. c. S. 267.

21 Ackerknecht, 1958, 1. c. S. 7. 
sie zeigt einerseits die skeptisch-abwartenden Züge der sonstigen Therapie, ja sie ist in ihrer Gesamthaltung häufig recht konservativ; die Kriegsbedingungen und der neue Lokalismus veranlassen sie andererseits mindestens vorübergehend zu einem therapeutischen Aktivismus, der allerdings auch in der inneren Medizin gelegentlich durchbricht. Neuerer in der Art eines Magendie hat sie nicht gehabt. Roux hatte darum wohl recht, wenn er die Periode 1800-1830 für die glänzendste in der französischen Chirurgie hielt ${ }^{22}$.

${ }^{22}$ Quarante années de pratique chirurgicale, Paris 1854, S. XIII. 